\title{
Mammography breast density: an effective supplemental modality for the precise grading of ultrasound BI-RADS 4 categories
}

\author{
Wei-Min Li ${ }^{1}$, Qiu-Wei Sun ${ }^{1}$, Xiao-Fang Fan ${ }^{1}$, Jun-Chao Zhang ${ }^{1}$, Ting Xu ${ }^{2}$, Qi-Qi Shen ${ }^{1}$, Lei Jia ${ }^{1}$ \\ ${ }^{1}$ Department of Ultrasonography, Affiliated Hospital of Jiangnan University, Wuxi, China; ${ }^{2}$ Department of Clinical and Research, Shenzhen Mindray \\ Biomedical Electronics Co., Ltd, Shenzhen, China \\ Contributions: (I) Conception and design: XF Fan, T Xu; (II) Administrative support: None; (III) Provision of study materials or patients: None; (IV) \\ Collection and assembly of data: XF Fan, T Xu; (V) Data analysis and interpretation: XF Fan, T Xu, WM Li, QW Sun; (VI) Manuscript writing: All \\ authors; (VII) Final approval of manuscript: All authors. \\ Correspondence to: Xiao-Fang Fan. Department of Ultrasonography, Affiliated Hospital of Jiangnan University, Wuxi 214000, China. \\ Email: fanxiaoll1@126.com.
}

Background: High breast density is significantly associated with an increased risk of breast diseases. Presently, suspected breast masses assessed as Breast Imaging-Reporting and Data System (BI-RADS) grade 4 provide a wide range of positive predictive values. Moreover, subcategories $(4 \mathrm{a}, 4 \mathrm{~b}$, and $4 \mathrm{c})$ are still under consideration as the diagnostic criteria are neither comprehensive nor objective. However, whether mammography breast density (MBD) has any impact on the accurate grading of BI-RADS 4 assessed by ultrasound (US) remains unknown.

Methods: A total of 1,086 women with 1,293 breast masses were included and assessed as BI-RADS 3-5 by US. The subcategories of MBD (from the ACR-a to the ACR-d group) were assessed by mammography according to the criteria of the American College of Radiology (ACR). The clinicopathological characteristics of these patients were reviewed retrospectively. The malignancy rates of breast masses among different subgroups assessed by BI-RADS were re-estimated with MBD.

Results: Almost all BI-RADS 3 masses were classified as benign and nearly all BI-RADS 5 masses were identified as malignant. Significant inverse associations between MBD and malignancy rates were detected between the BI-RADS $4 \mathrm{a}$ and BI-RADS $4 \mathrm{~b}$ groups. Moreover, malignancy rates decreased significantly from ACR-a to ACR-d for BI-RADS $4 a$ and $4 b$ breast lesions $(\mathrm{P}<0.001)$. However, this trend was not observed in BI-RADS 4c breast lesions.

Conclusions: MBD could serve as a crucial factor for the accurate grading of BI-RADS 4 lesions assessed by US. We strongly recommend the adoption of the MBD as a possible supplemental screening modality for US. Furthermore, it is equally beneficial for accurate risk assessment and screening recommendations based on MBD.

Keywords: Mammographic breast density (MBD); BI-RADS 4; ultrasound (US)

Submitted Apr 02, 2021. Accepted for publication Jun 17, 2021.

doi: $10.21037 /$ gs-21-313

View this article at: https://dx.doi.org/10.21037/gs-21-313

\section{Introduction}

Worldwide, breast cancer is one of the most frequent malignancies diagnosed among women, impacting 1.67 million female patients each year. It is also the leading cause of cancer-related mortality among women in countries with a low level of development (1). Thus, strengthening the awareness of the benefits of screening and early detection is highly desirable. Screening mammography remains the most useful diagnostic modality for the early detection of breast cancer in asymptomatic patients, which can also assess breast density. Mammographic breast density is an established risk marker for breast cancer and is 
visually assessed by radiologists in routine mammogram image reading, using four qualitative Breast Imaging and Reporting Data System (BI-RADS) breast density categories. Mammographic breast density (MBD) is one of the established risk factors for breast cancer. Furthermore, elevated MBD has been consistently associated with an increased risk of breast cancer (2-4). However, high MBD can "mask" an emerging tumor on standard mammography and therefore requires additional supplemental diagnostic tools $(5,6)$. Ultrasound (US) screening is now receiving increased attention as a proven supplemental screening tool to differentiate between benign and malignant breast lesions.

Breast Imaging-Reporting and Data System (BI-RADS) is a widely accepted risk assessment and quality assurance tool in mammography, US, or magnetic resonance imaging. Usually, the prediction of malignancy for BIRADS category 3 is $0 \%$ to $2 \%$ and nearly $95 \%$ or higher for BI-RADS 5. As BI-RADS 4 is the least predictable, this category is sub-divided into 3 subgroups, including $4 \mathrm{a}$ (low), 4b (medium), and 4c (substantial). The positive predictive values for BI-RADS $4 \mathrm{a}$ were defined as $2-10 \%$, BI-RADS $4 \mathrm{~b}$ as $10-50 \%$, and BI-RADS $4 \mathrm{c}$ as $50-95 \%$ (7). BI-RADS 4 masses with dense breasts have a moderately increased risk of breast cancer, and dense breasts substantially reduced the sensitivity of mammography to detect malignancy $(4,6)$. As we known, it is particularly difficult for radiologists to consistently distinguish the two most common and most variably assigned BI-RADS categories. MBD plays an important role in breast cancer, so it may paly an important role in BI-RADS. Meanwhile, So our study aimed to evaluate the clinical utility of MBD in tailoring the precise sub-categorization of BI-RADS-US 4. Furthermore, the utility of MBD in predicting the rate of malignancy in women with BI-RADS-US 4 remains unknown. Therefore, the present study aimed to evaluate the clinical utility of MBD in tailoring the precise sub-categorization of BIRADS-US 4. We present the following article in accordance with the STROBE reporting checklist (available at https:// dx.doi.org/10.21037/gs-21-313).

\section{Methods}

\section{Selection of patients}

All procedures performed in this study involving human participants were in accordance with the Declaration of Helsinki (as revised in 2013). The study was approved by the Ethics Committee of Affiliated Hospital of Jiangnan University. Individual consent for this retrospective analysis was waived. From October 2015 to June 2017, a total of 1,086 women with 1,293 breast masses retrospectively assessed as BI-RADS 3-5 by US were included in the study. The mammographic data were also acquired. The clinical and pathological records of these patients were retrieved from the archive system. The data, including age, body mass index (BMI), cancer rates, mammograms, US prints, and reports, were carefully reviewed.

\section{Ultrasonography examination}

Superficial gray-scale ultrasonography examination of the breast was performed using high-resolution US equipment, the Resona 7 pro US real-time unit with a $12 \mathrm{MHz}$ lineararray transducer. In the case of a mass, the type of lesion (solid, complex, cystic, or pure cystic), the location, size, echogenicity, contour, and acoustic features were evaluated, and the presence of axillary lymph nodes was noted. Both breasts were systematically examined with overlapping scans.

\section{Retrospective image review}

All US characteristics of the breast masses were retrospectively reviewed and re-evaluated by BI-RADS according to the 5 th edition of the American Academy of Radiology (ACR) (7). Breast masses were selected for further evaluation if assessments provided by 2 ultrasonic physicians were inconsistent. The ultrasonic physicians were blinded to the final pathological diagnosis of all breast masses. MBD was measured using the 4 density categories of the ACR BI-RADS: mostly fatty with less than $25 \%$ of fibroglandular tissue (ACR-a), scattered fibroglandular tissue ranging from $25 \%$ to $50 \%$ (ACR-b), heterogeneously dense tissue ranging from $51 \%$ to $75 \%$ (ACR-c), and fibroglandular tissue of greater than 75\% (ACR-d) (8).

\section{Hematoxylin and eosin (HE) staining}

The tissue of breast tumor was immersed in $4 \%$ paraformaldehyde for $4 \mathrm{~h}$, and transferred to $70 \%$ ethanol. Individual lobes of breast tumor were placed in processing cassettes, dehydrated through a serial alcohol gradient, and embedded in paraffin wax blocks. Before immunostaining, 5-um-thick lung tissue sections were dewaxed in xylene, rehydrated through decreasing concentrations of ethanol, 
Table 1 General information of the 1,293 breast masses in 1,086 patients

\begin{tabular}{|c|c|c|c|c|c|}
\hline Characteristics & ACR-a & ACR-b & ACR-C & ACR-d & $P$ value \\
\hline Number of masses & 89 & 377 & 582 & 245 & \\
\hline Mean age $\pm S D$, years & $57.62 \pm 15.38$ & $51.57 \pm 17.36$ & $43.57 \pm 17.18$ & $35.93 \pm 14.29$ & $<0.0001$ \\
\hline $\mathrm{BMI}, \mathrm{kg} / \mathrm{m}^{2}$ & $28.86 \pm 4.08$ & $25.41 \pm 3.72$ & $22.64 \pm 3.63$ & $20.62 \pm 4.13$ & $<0.0001$ \\
\hline Mean size $(\mathrm{cm}) \pm \mathrm{SD}$ & $1.93 \pm 0.93$ & $1.83 \pm 0.78$ & $1.84 \pm 0.92$ & $1.89 \pm 0.79$ & 0.135 \\
\hline Family history of breast cancer & 7 & 8 & 13 & 5 & 0.051 \\
\hline Malignancy rate (\%) & 70.79 & 40.85 & 37.63 & 22.86 & $<0.0001$ \\
\hline
\end{tabular}

ACR, American College of Radiology; SD, standard deviation; BMI, body mass index.

and washed in PBS. And then stained with hematoxylin and eosin (H\&E). After staining, sections were dehydrated through increasing concentrations of ethanol and xylene.

\section{Statistical analysis}

Data were represented as frequencies and percentages for categorical variables, and means and standard deviations for continuous variables. PASS was used to calculate the study size. Associations of MBD with clinical variables were assessed using chi-square tests of significance. Fisher's exact test was used to establish the association between BI-RADS category and malignancy rate. Malignancy rates across MBD were assessed using the linear-by-linear association test. All statistical analyses were performed using SPSS version 18.0 for Windows (SPSS Inc., Chicago, IL, USA). P values $<0.05$ were considered statistically significant.

\section{Results}

\section{Baseline data in clinical trials}

A total of 1,086 women with 1,293 breast masses were retrospectively reviewed as BI-RADS $3-5$ by US. Of these, $38.05 \%(492 / 1,293)$ of the lesions were malignant. The median age of the cohort was 45 years old (ranged from 18 to 85 years). Basic characteristics of the patients including age, BMI, and malignancy rates were recorded among different MBD groups and represented in Table 1.

Older age, higher BMI, and higher malignancy rates were significantly associated with lower MBD, whereas younger age, lower BMI, and lower malignancy rates were significantly associated with higher MBD (Table 1).
However, there was no significant difference between tumor size, tumor margin, family history of breast cancer, and MBD subtypes (Table 1).

\section{The association between $M B D$ and malignancy rates among different BI-RADS-US groups}

Moreover, the linear-by-linear association test revealed significantly decreased malignancy rates across MBD groups (from the ACR-a group to the ACR-d group) for both BIRADS $4 \mathrm{a}$ and $4 \mathrm{~b}$ categories (Figure 1) $(\mathrm{P}<0.001)$. However, this trend was not observed in BI-RADS 3, 4c, and 5 categories. Almost all BI-RADS 3 masses were identified as benign, and nearly all BI-RADS $4 \mathrm{c}$ and 5 masses were identified as malignant. BI-RADS-US 4 breast lesions in different MBD subtypes are shown in Figures 2,3,4.

Taken together, these findings indicate that MBD may be a potential candidate tool in tailoring the precise grading of BI-RADS 4 categories, particularly for $4 \mathrm{a}$ and $4 \mathrm{~b}$ subgroups.

\section{Discussion}

MBD reflects the proportion of the breast occupied by radiologically dense fibroglandular tissue. Moreover, an elevated mammographic density is an established risk factor for breast cancer. Additionally, healthy women with higher MBD may have an increased risk of developing breast cancer $(9,10)$.

The BI-RADS-US classification, as revised by the ACR in 2013, has proven to be highly useful in differentiating between benign and malignant lesions. Consistent with previous findings $(11,12)$, we found that breast masses in 


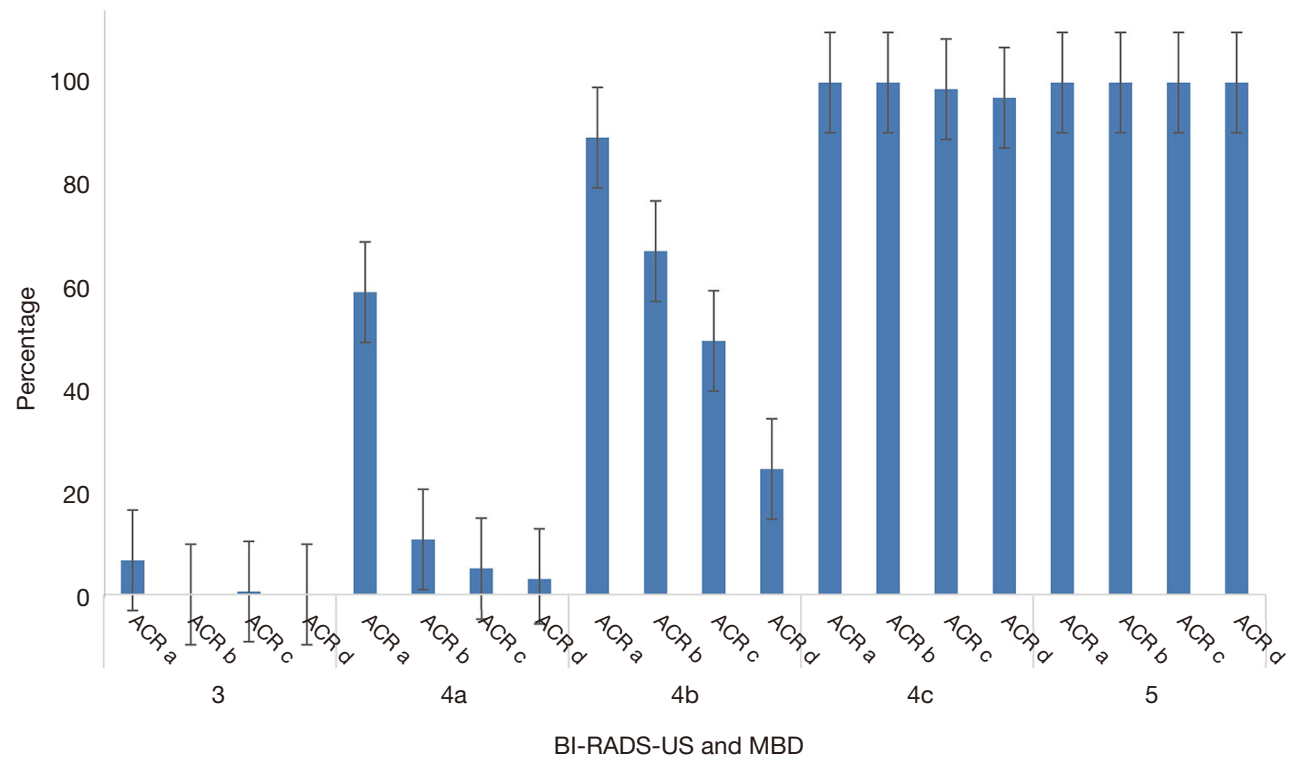

Figure 1 The association between MBD and malignancy rates among different BI-RADS-US groups. The linear-by-linear association test revealed significantly decreased $(\mathrm{P}<0.001)$ malignancy rates across MBD groups (from the ACR-a to the ACR-d group) for BI-RADS-US $4 \mathrm{a}$ and $4 \mathrm{~b}$ categories. However, this trend was not observed in BI-RADS-US 3, 4c, and 5 categories. ACR, American College of Radiology; BIRADS, Breast Imaging-Reporting and Data System; MBD, mammography breast density; US, ultrasound.

patients with BI-RADS 3 exhibited a lower malignancy rate $(0.54 \%)$. However, higher malignancy rates were observed in patients with BI-RADS 5 (100\%). Notably, BI-RADS-US category $4(4 \mathrm{a}, 4 \mathrm{~b}$, and $4 \mathrm{c})$ was advantageous in predicting malignancy. However, it is particularly challenging to categorize it into different sub-classes. Therefore, objective and accurate sub-classification is highly desirable (13-15).

Studies have indicated that both MBD and BI-RADS-US were independent risk factors for breast cancer, particularly in women with suspected breast lesions $(9,11)$. However, there is a paucity of studies considering both MBD and BIRADS-US together in predicting the possibility of breast cancer, and particularly for accurate grading of BI-RADS category 4 . The key point of present research suggest that MBD play an important role in breast cancer, and in different MBD group, the linear-by-linear association test revealed significantly decreased malignancy rates across MBD groups (from the ACR-a group to the ACR-d group) for both BI-RADS $4 \mathrm{a}$ and $4 \mathrm{~b}$ categories.

In the present study, we found that the malignancy rates (in BI-RADS-US 4a and 4b, but not in 4c) were significantly decreased with the increased MBD. Moreover, lower MBD appeared to increase the BI-RADS grade of breast tumors. The differences between our findings and those reported earlier are as follows. First, only patients with breast masses were included in our study while other studies enrolled patients without breast lumps $(3,9)$. Second, as a substitute for mammographic evaluation that was primarily used in other studies, we considered US in order to avoid the effect of dense breasts on the accurate grading of breast lesions (16). Third, compared to Western women whose breasts contain more fat, patients enrolled in our study were all Asian women whose breasts have a lower proportion of fat $(17,18)$. Finally, breast masses of BI-RADS-US $4 \mathrm{c}$ and 5 exhibited a higher tendency for malignancy, and most of them were confirmed to be malignant by histopathology in our study.

To the best of our knowledge, this is the first study to indicate that the higher the MBD, the higher the possibility of malignancy in BI-RADS-US $4 \mathrm{a}$ and $4 \mathrm{~b}$ groups. In agreement with other studies $(19,20)$, our results also suggested that higher MBD was associated with younger age and lower BMI. Thus, age and BMI are important factors affecting both the MBD and malignancy rates. Similar to age and BMI, MBD could be an important supplemental modality for BI-RADS-US in predicting the possibility of breast cancer in BI-RADS 4 lesions.

Furthermore, several adipocytokines and estrogen are produced by adipose tissue, which may function in 

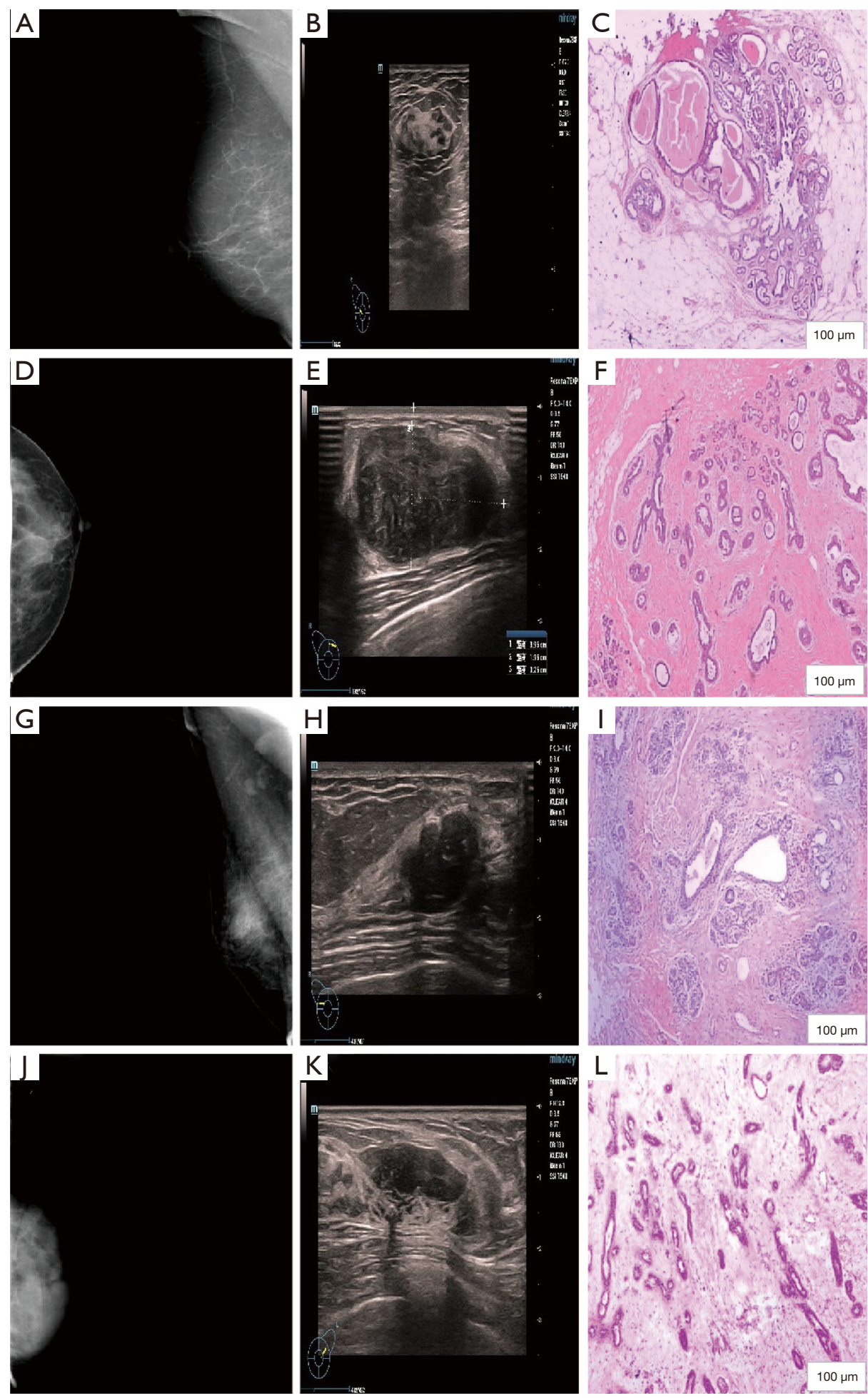

Figure 2 BI-RADS 4a breast masses assessed by ultrasound in different MBD groups. Breast masses with ACR-a (A), ACR-b (D), ACR-c (G), and ACR-d (J) on mammography were depicted by representative US (B, E, H, and K) and histopathology of HE staining (C, F, I, and L). ACR, American College of Radiology; BI-RADS, Breast Imaging-Reporting and Data System; MBD, mammography breast density; US, ultrasound. 

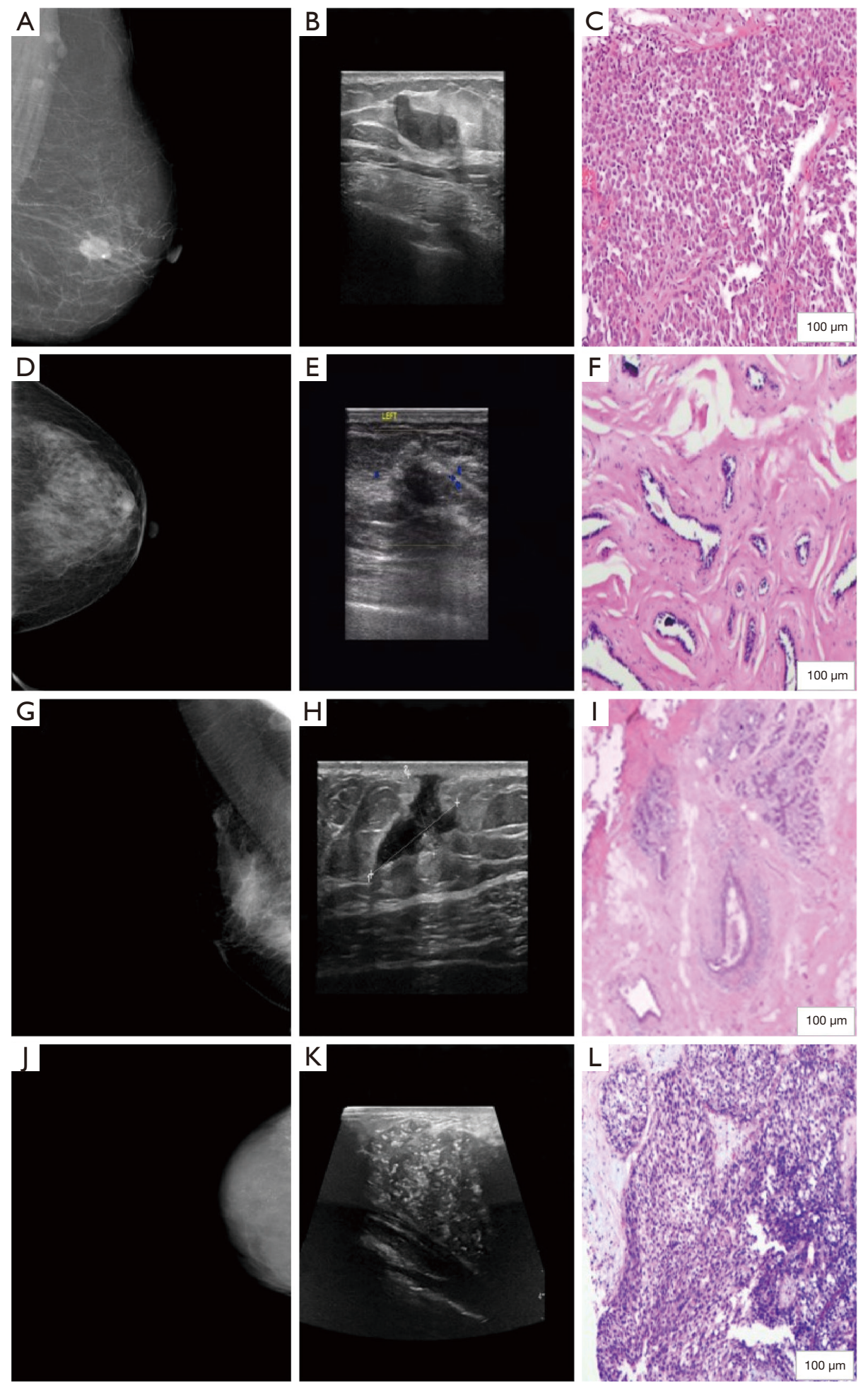

Figure 3 BI-RADS 4b breast masses assessed by ultrasound in different MBD groups. Breast masses with ACR-a (A), ACR-b (D), ACR-c (G), and ACR-d (J) on mammography were depicted by representative US (B, E, H, and K) and histopathology of HE staining (C, F, I, and L). ACR, American College of Radiology; BI-RADS, Breast Imaging-Reporting and Data System; MBD, mammography breast density; US, ultrasound. 

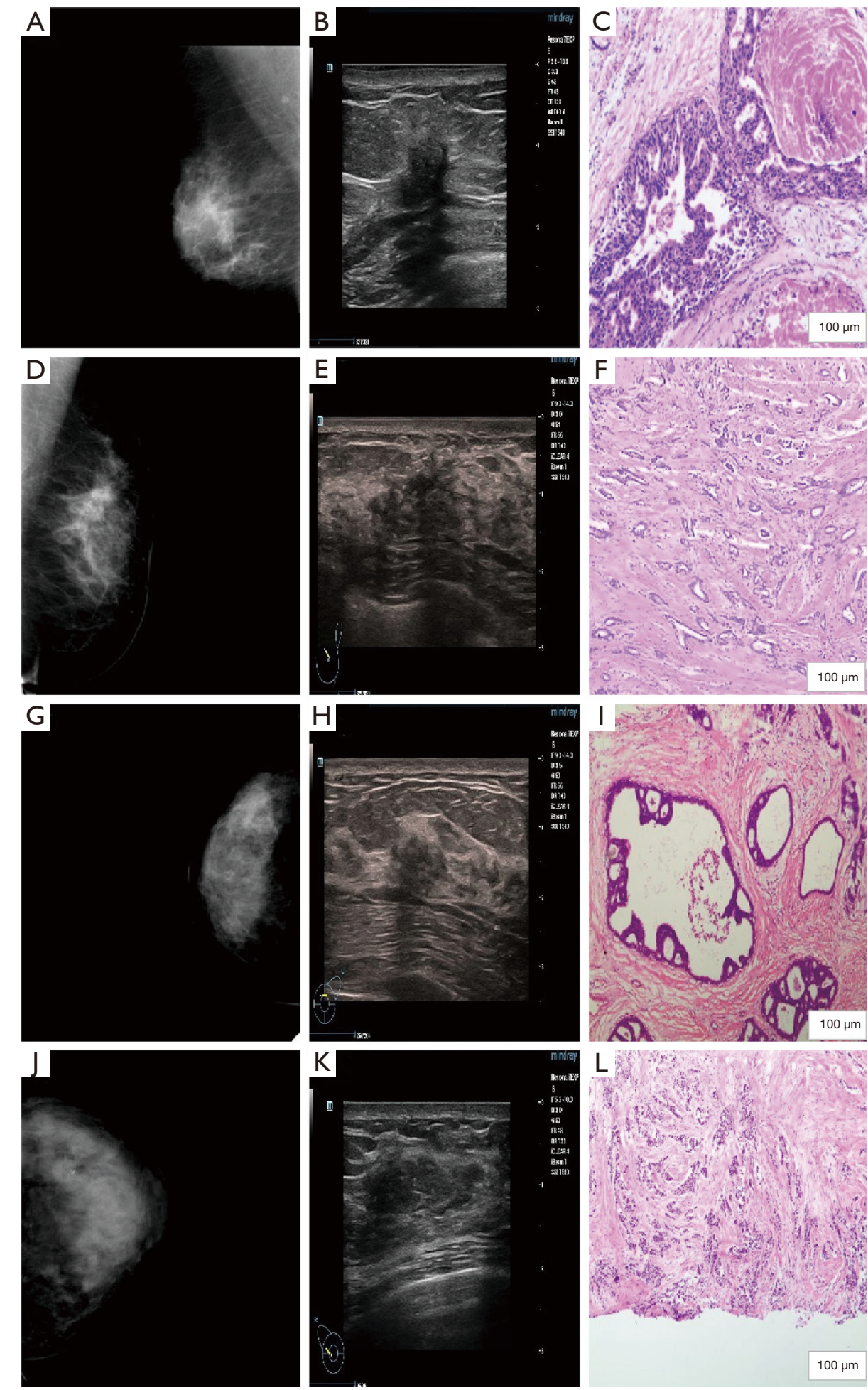

Figure 4 BI-RADS 4c breast masses assessed by ultrasound in different MBD groups. Breast masses with ACR-a (A), ACR-b (D), ACR-c $(\mathrm{G})$, and ACR-d (J) on mammography were shown by representative US (B, E, H, and K) and histopathology of HE staining (C, F, I, and L). ACR, American College of Radiology; BI-RADS, Breast Imaging-Reporting and Data System; MBD, mammography breast density; US, ultrasound. 
conjunction with hormones and growth factors to provide a favorable microenvironment for the occurrence and development of breast cancer (21-23). This could also be the reason for the higher malignancy rate in ACR-a breasts. However, there is a paucity of literature on the association between total adipose tissue and breast cancer. Thus, suspicious breast masses with lower MBD could prompt higher chances of malignancy when there are diagnostic disparities, particularly in the fatty breast (ACR-a).

Consequently, in breast imaging diagnostics, when there is ambiguity between BI-RADS-US $4 \mathrm{a}$ or $4 \mathrm{~b}$, it may be beneficial to assign the lesion a higher category if it belongs to fatty breast tissue (ACR-a). In contrast, BI-RADS-US 4a or $4 \mathrm{~b}$ with dense breast tissue (ACR-d) are most likely to account for lower malignancy rates. This may be attributed to the hyperplasia of the mammary gland. Breast-related diseases are frequently diagnosed in women aged from 20 to 50 years old due to hyperplasia of the mammary gland, mostly occurring in ACR-d breasts, which has the potential $(5-10 \%)$ to develop into breast cancer (24). However, it may not be malignant at the early stages.

Earlier reports have also demonstrated that breast masses in women with higher MBD exhibited a higher risk of developing into malignant tumors (25). However, in our study, breast masses in women with lower MBD had an increased probability of malignancy than those with higher MBD. Thus, MBD as a supplemental modality could accurately and consistently expedite BI-RADS-US subcategorization (particularly of BI-RADS $4 a$ and $4 b$ ) for precise clinical decision making.

\section{Limitations}

The patients enrolled in this study are come from a hospital in China. The malignancy rates of breast mass may be different in different breast density due to different races. Meanwhile, the sample size is limited in this paper. The association between MBD and malignancy rates among different BI-RADS-US groups may be different to some degree.

\section{Conclusions}

MBD could be an important tool to amend the accurate grading of BI-RADS 4 assessed by US. We strongly suggest the adoption of the MBD as a supplemental modality to facilitate accurate risk assessment and precise screening recommendations for BI-RADS-US 4 categories.

\section{Acknowledgments}

Funding: None.

\section{Footnote}

Reporting Checklist: The authors have completed the STROBE reporting checklist. Available at https://dx.doi. org/10.21037/gs-21-313

Data Sharing Statement: Available at https://dx.doi. org/10.21037/gs-21-313

Conflicts of Interest: All authors have completed the ICMJE uniform disclosure form (available at https://dx.doi. org/10.21037/gs-21-313). Ting Xu reports that she is from Shenzhen Mindray Biomedical Electronics Co., Ltd. The other authors have no conflicts of interest to declare.

Ethical Statement: The authors are accountable for all aspects of the work in ensuring that questions related to the accuracy or integrity of any part of the work are appropriately investigated and resolved. All procedures performed in this study involving human participants were in accordance with the Declaration of Helsinki (as revised in 2013). The study was approved by the Ethics Committee of Affiliated Hospital of Jiangnan University. Individual consent for this retrospective analysis was waived.

Open Access Statement: This is an Open Access article distributed in accordance with the Creative Commons Attribution-NonCommercial-NoDerivs 4.0 International License (CC BY-NC-ND 4.0), which permits the noncommercial replication and distribution of the article with the strict proviso that no changes or edits are made and the original work is properly cited (including links to both the formal publication through the relevant DOI and the license). See: https://creativecommons.org/licenses/by-nc-nd/4.0/.

\section{References}

1. Ghoncheh M, Pournamdar Z, Salehiniya H. Incidence and Mortality and Epidemiology of Breast Cancer in the World. Asian Pac J Cancer Prev 2016;17:43-6.

2. Nazari SS, Mukherjee P. An overview of mammographic density and its association with breast cancer. Breast Cancer 2018;25:259-67.

3. Duffy SW, Morrish OWE, Allgood PC, et al. 
Mammographic density and breast cancer risk in breast screening assessment cases and women with a family history of breast cancer. Eur J Cancer 2018;88:48-56.

4. Huo CW, Chew GL, Britt KL, et al. Mammographic density-a review on the current understanding of its association with breast cancer. Breast Cancer Res Treat 2014;144:479-502.

5. Ang ZZ, Rawashdeh MA, Heard R, et al. Classification of normal screening mammograms is strongly influenced by perceived mammographic breast density. J Med Imaging Radiat Oncol 2017;61:461-9.

6. Moshina N, Ursin G, Roman M, et al. Positive predictive values by mammographic density and screening mode in the Norwegian Breast Cancer Screening Program. Eur J Radiol 2016;85:248-54.

7. Mercado CL. BI-RADS update. Radiol Clin North Am 2014;52:481-7.

8. Destounis S, Arieno A, Morgan R, et al. Qualitative Versus Quantitative Mammographic Breast Density Assessment: Applications for the US and Abroad. Diagnostics (Basel) 2017;7:1-33.

9. Rice MS, Bertrand KA, VanderWeele TJ, et al. Mammographic density and breast cancer risk: a mediation analysis. Breast Cancer Res 2016;18:94-106.

10. Habel LA, Lipson JA, Achacoso N, et al. Case-control study of mammographic density and breast cancer risk using processed digital mammograms. Breast Cancer Res 2016;18:53-9.

11. Raza S, Chikarmane SA, Neilsen SS, et al. BI-RADS 3, 4, and 5 lesions: value of US in management--follow-up and outcome. Radiology 2008;248:773-81.

12. Kim EK, Ko KH, Oh KK, et al. Clinical application of the BI-RADS final assessment to breast sonography in conjunction with mammography. AJR Am J Roentgenol 2008;190:1209-15.

13. Spinelli Varella MA, Teixeira da Cruz J, Rauber A, et al. Role of BI-RADS Ultrasound Subcategories 4A to $4 \mathrm{C}$ in Predicting Breast Cancer. Clin Breast Cancer 2018;18:e507-11.

14. Leng X, Huang G, Yao L, et al. Role of multi-mode ultrasound in the diagnosis of level 4 BI-RADS breast lesions and Logistic regression model. Int J Clin Exp Med 2015;8:15889-99.

15. Elverici E, Barca AN, Aktas H, et al. Nonpalpable BIRADS 4 breast lesions: sonographic findings and pathology correlation. Diagn Interv Radiol 2015;21:189-94.
16. Sanabria SJ, Goksel O, Martini K, et al. Breast-density assessment with hand-held ultrasound: A novel biomarker to assess breast cancer risk and to tailor screening? Eur Radiol 2018;28:3165-75.

17. Manning M, Purrington K, Penner L, et al. Between-race differences in the effects of breast density information and information about new imaging technology on breasthealth decision-making. Patient Education Counseling 2016;99:1002-10.

18. Bae JM, Kim EH. Breast Density and Risk of Breast Cancer in Asian Women: A Meta-analysis of Observational Studies. J Prev Med Public Health 2016;49:367-75.

19. Hack CC, Emons J, Jud SM, et al. Association between mammographic density and pregnancies relative to age and BMI: a breast cancer case-only analysis. Breast Cancer Res Treat 2017;166:701-8.

20. Andersen ZJ, Baker JL, Bihrmann K, et al. Birth weight, childhood body mass index, and height in relation to mammographic density and breast cancer: a register-based cohort study. Breast Cancer Res 2014;16:R4-14.

21. Pallua N, Kim BS. Commentary on: Interaction Between Breast Cancer Cells and Adipose Tissue Cells Derived from Fat Grafting. Aesthet Surg J 2016;36:364-5.

22. Massa M, Gasparini S, Baldelli I, et al. Interaction Between Breast Cancer Cells and Adipose Tissue Cells Derived from Fat Grafting. Aesthet Surg J 2016;36:358-63.

23. Vona-Davis L, Rose DP. Angiogenesis, adipokines and breast cancer. Cytokine Growth Factor Rev 2009;20:193-201.

24. Lv P, Chong Y, Zou H, et al. A Study of Using Massage Therapy Accompanied with Stretching Exercise for Rehabilitation of Mammary Gland Hyperplasia. BioMed Res Int 2016;2016:9426167.

25. Tice JA, Miglioretti DL, Li CS, et al. Breast Density and Benign Breast Disease: Risk Assessment to Identify Women at High Risk of Breast Cancer. J Clin Oncol 2015;33:3137-43.

(English Language Editor: C. Betlazar-Maseh)

Cite this article as: $\mathrm{Li} W M$, Sun QW, Fan XF, Zhang JC, Xu T, Shen QQ, Jia L. Mammography breast density: an effective supplemental modality for the precise grading of ultrasound BIRADS 4 categories. Gland Surg 2021;10(6):2010-2018. doi: $10.21037 / \mathrm{gs}-21-313$ 